\title{
In Silico Guidance for In Vitro Androgen and Glucocorticoid Receptor ToxCast Assays
}

Timothy E. H. Allen, ${ }^{1,2}$ Mark D. Nelms, ${ }^{3,4}$ Stephen W. Edwards, ${ }^{4}$ Jonathan M.

Goodman, ${ }^{* 1}$ Steve Gutsell, ${ }^{5}$ and Paul J. Russel/ ${ }^{5}$

${ }^{1}$ Centre for Molecular Informatics, Department of Chemistry, University of Cambridge,

Lensfield Road, Cambridge, CB2 1EW, United Kingdom

2MRC Toxicology Unit, University of Cambridge, Hodgkin Building, Lancaster Road,

Leicester, LE1 7HB, United Kingdom

${ }^{3}$ Oak Ridge Institute for Science and Education, Oak Ridge, TN 37830, USA

${ }^{4}$ Integrated Systems Toxicology Division, National Health and Environmental Effects

Research Laboratory, Office of Research and Development, U.S. Environmental

Protection Agency, Research Triangle Park, Durham, NC 27709, USA

${ }^{5}$ Unilever Safety and Environmental Assurance Centre, Colworth Science Park,

Sharnbrook, Bedfordshire, MK44 1LQ, United Kingdom 
Supporting Information

SMILES strings for the structural alerts developed in this work

S2-S5

Performance statistics for the structural alerts developed in this work

S6S10

\section{Androgen Receptor}

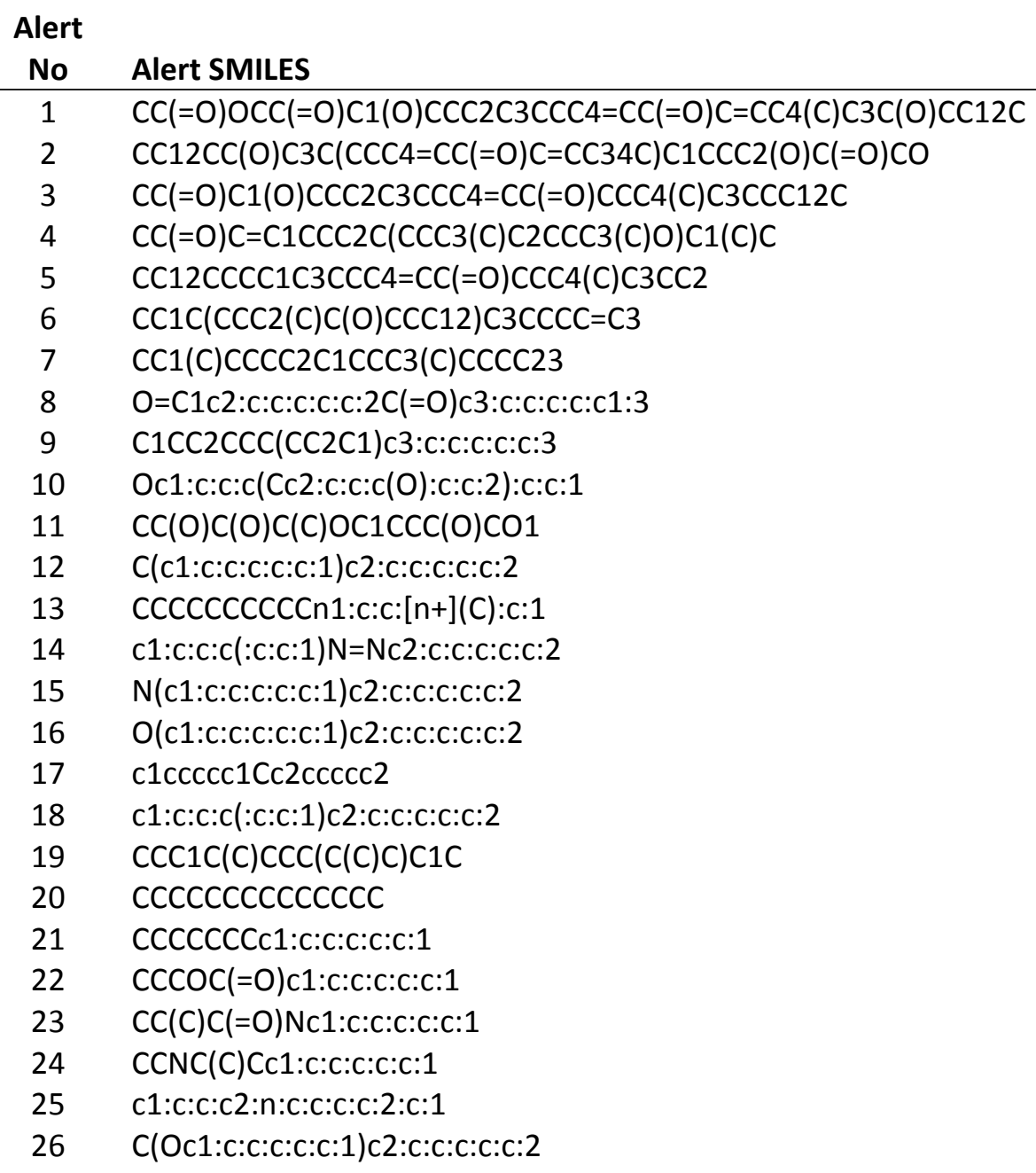




\begin{tabular}{|c|c|}
\hline 27 & $\mathrm{OC}(=\mathrm{O}) \mathrm{C}(=\mathrm{C}) \mathrm{Cc} 1: \mathrm{c}: \mathrm{c}: \mathrm{c}: \mathrm{c}: \mathrm{c}: 1$ \\
\hline 28 & CCN(CC)c1:c:c:c:c:c:1 \\
\hline 29 & CCCNCc1:c:c:c:c:c:1 \\
\hline 30 & cсcсcсcсcсcс \\
\hline 31 & C(Cc1:c:c:c:c:c:1)c2:c:c:c:c:c:2 \\
\hline 32 & $\mathrm{O}(\mathrm{c} 1: c: c: c: c: c: 1) c 2: c: c: c: c: n: 2$ \\
\hline 33 & CCCCc1:c:c:c:c:c:1 \\
\hline 34 & CC(=0)Nc1:c:c:c:c:c:1 \\
\hline 35 & $\mathrm{O}=\mathrm{CC}=\mathrm{Cc} 1: \mathrm{c}: \mathrm{c}: \mathrm{c}: \mathrm{c}: \mathrm{c}: 1$ \\
\hline 36 & CNCNc1:c:c:c:c:c:1 \\
\hline 37 & COC(=0)Nc1:c:c:c:c:c:1 \\
\hline 38 & CCNCCc1:c:c:c:c:c:1 \\
\hline 39 & CCCOc1:c:c:c:c:c:1 \\
\hline 40 & $\operatorname{coc}(=0) c 1: c: c: c: c: c: 1$ \\
\hline 41 & COc1:c:c(C):c:c:c:10 \\
\hline 42 & $\mathrm{O}=\mathrm{S}(=0) \mathrm{c} 1: \mathrm{c}: \mathrm{c}: \mathrm{c}: \mathrm{c}: \mathrm{c}: 1$ \\
\hline 43 & {$[0-][N+](=0) c 1: c: c: c: c: c: 1$} \\
\hline 44 & CCOCCc1:c:c:c:c:c:1 \\
\hline 45 & $\mathrm{CC}(\mathrm{C})(\mathrm{C}) \mathrm{c} 1: \mathrm{c}: \mathrm{c}: \mathrm{c}: \mathrm{c}: \mathrm{c}: 1$ \\
\hline 46 & {$[\mathrm{nH}] 1: \mathrm{c} 2: \mathrm{c}: \mathrm{c}: \mathrm{c}: \mathrm{c}: \mathrm{c}: 2: \mathrm{c}: \mathrm{c}: 1$} \\
\hline 47 & 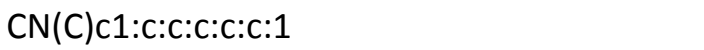 \\
\hline 48 & CCOc1:c:c:c:c:c:1 \\
\hline 49 & Cc1:c:c:c(Cl):c:c:1Cl \\
\hline 50 & $\operatorname{ccoc}(=0) C(C C) C C$ \\
\hline 51 & $\operatorname{ccc} 1 \operatorname{CcCC}(\mathrm{C}) \mathrm{C} 1$ \\
\hline 52 & COc1:c:c:c:c:c:1 \\
\hline 53 & $\operatorname{cccc}[\mathrm{N}+](\mathrm{C})(\mathrm{CCCC}) \mathrm{CCCC}$ \\
\hline 54 & CCCC[n+]1:c:c:c:c:c:1 \\
\hline 55 & c1:c:c:c2:[nH]:c:n:c:2:c:1 \\
\hline 56 & c1:c:c:c2:s:c:n:c:2:c:1 \\
\hline 57 & $\operatorname{ccsc} 1 \mathrm{CC}(=0) \mathrm{N} 1 \mathrm{CC}$ \\
\hline 58 & O=Cc1:c:c:c:c:c:1 \\
\hline 59 & $\operatorname{ccccc}(\mathrm{CC}) \mathrm{CO}$ \\
\hline 60 & Clc1:c:c:c:c(Cl):c:1 \\
\hline 61 & OCC10сCC10 \\
\hline 62 & Cc1:c:c:c:c:c:1N \\
\hline 63 & $\mathrm{CCOP}(=\mathrm{S})(\mathrm{OC}) \mathrm{OC}$ \\
\hline 64 & CCn1:c:c:[n+](C):c:1 \\
\hline 65 & Cc1:c:c:c(C):c:c:1 \\
\hline 66 & Cc1:c:c:c:c:c:1 \\
\hline 67 & c1:c:c:c(:c:c:1)[Bi](c2:c:c:c:c:c:2)c3:c:c:c:c:c:3 \\
\hline 68 & $\mathrm{C}[\mathrm{SiH}](\mathrm{c} 1: \mathrm{c}: \mathrm{c}: \mathrm{c}: \mathrm{c}: \mathrm{c}: 1) \mathrm{c} 2: \mathrm{c}: \mathrm{c}: \mathrm{c}: \mathrm{c}: \mathrm{c}: 2$ \\
\hline 69 & $\mathrm{CN}(\mathrm{C}) \mathrm{C}(=\mathrm{S}) \mathrm{SSC}(=\mathrm{S}) \mathrm{N}(\mathrm{C}) \mathrm{C}$ \\
\hline 70 & c1:c:c:c2:c:n:n:c:c:2:c:1 \\
\hline 71 & $\operatorname{CNCN} 1 \mathrm{CCN}(\mathrm{C}) \mathrm{CC} 1$ \\
\hline 72 & $\operatorname{CCCN}(\mathrm{C}) \mathrm{CCCNC}$ \\
\hline 73 & $\mathrm{O}=\mathrm{C} 1 \mathrm{CCCCCN} 1$ \\
\hline 74 & $\mathrm{CCCC}[\mathrm{Sn}] \mathrm{CCCC}$ \\
\hline
\end{tabular}




$\begin{array}{ll}75 & \text { CC1CCCC=C1C } \\ 76 & \text { OC(=O)CC(S)C(=O)O } \\ 77 & \text { Nc1:c:c:c:c(Cl):c:1 } \\ 78 & \text { CC[n+]1:c:c:c:c:c:1 } \\ 79 & \text { CCCCCCCC } \\ 80 & {[0-][n+] 1: c: c: c: c: c: 1} \\ 81 & \text { CCCCC=C(C)C } \\ 82 & \text { CCCC(CC)C=O } \\ 83 & \text { Nc1:c:c:c:c:c:1 } \\ 84 & \text { c1:c:c:c:c:c:1 } \\ 85 & \text { CC(O)CCC=O } \\ 86 & \text { COCCC(=O)O } \\ 87 & \text { Cc1:0:c:c:c:1 }\end{array}$

\section{Glucocorticoid Receptor}

\begin{tabular}{|c|c|}
\hline $\begin{array}{c}\text { Alert } \\
\text { No }\end{array}$ & Alert SMILES \\
\hline 1 & $\mathrm{CC} 1(\mathrm{C}) \mathrm{OC} 2 \mathrm{CC} 3 \mathrm{C} 4 \mathrm{CCC} 5=\mathrm{CC}(=\mathrm{O}) \mathrm{C}=\mathrm{CC} 5(\mathrm{C}) \mathrm{C} 4 \mathrm{C}(\mathrm{O}) \mathrm{CC} 3(\mathrm{C}) \mathrm{C} 2(\mathrm{O} 1) \mathrm{C}(=\mathrm{O}) \mathrm{CO}$ \\
\hline 2 & $\mathrm{CC}(=0) \mathrm{OCC}(=0) \mathrm{C} 1(\mathrm{O}) \mathrm{CCC} 2 \mathrm{C} 3 \mathrm{CCC} 4=\mathrm{CC}(=0) \mathrm{C}=\mathrm{CC} 4(\mathrm{C}) \mathrm{C} 3(\mathrm{~F}) \mathrm{C}(\mathrm{O}) \mathrm{CC} 12 \mathrm{C}$ \\
\hline 3 & $C C(=0) 0 C C(=0) C 1(0) C C C 2 C 3 C C C 4=C C(=0) C=C C 4(C) C 3 C(0) C C 12 C$ \\
\hline 4 & $\mathrm{CCC}(=0) \mathrm{OC} 1(\mathrm{C}=0) \mathrm{C}(\mathrm{C}) \mathrm{CC} 2 \mathrm{C} 3 \mathrm{CCC} 4=\mathrm{CC}(=\mathrm{O}) \mathrm{C}=\mathrm{CC} 4(\mathrm{C}) \mathrm{C} 3(\mathrm{~F}) \mathrm{CCC} 12 \mathrm{C}$ \\
\hline 5 & $\mathrm{CC} 12 \mathrm{CC}(0) \mathrm{C} 3 \mathrm{C}(\mathrm{CCC} 4=\mathrm{CC}(=0) \mathrm{C}=\mathrm{CC} 34 \mathrm{C}) \mathrm{C} 1 \mathrm{CCC} 2(0) \mathrm{C}(=0) \mathrm{CO}$ \\
\hline 6 & $\operatorname{cc} 12 \mathrm{CCC}(=0) \mathrm{C}=\mathrm{C} 1 \mathrm{CcC} 3 \mathrm{C} 4 \mathrm{CcC}(\mathrm{O})(\mathrm{C}(=\mathrm{O}) \mathrm{CO}) \mathrm{C} 4(\mathrm{C}) \mathrm{CC}(\mathrm{O}) \mathrm{C} 23$ \\
\hline 7 & 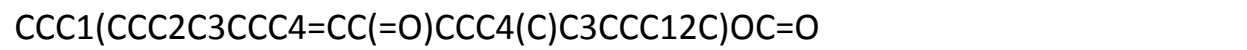 \\
\hline \multirow[t]{2}{*}{8} & $\mathrm{CC}(=0) \mathrm{C} 1(0) \mathrm{CCC} 2 \mathrm{C} 3 \mathrm{C}=\mathrm{CC} 4=\mathrm{CC}(=0) \mathrm{CCC} 4(\mathrm{C}) \mathrm{C} 3 \mathrm{CCC} 12 \mathrm{C}$ \\
\hline & COc1:c:c:c:c2C(=0)c3:c(O):c4CC(O)(CC(OC5CC(N)C(O)C(C)O5)c:4:c(O):c:3C(=O)c: \\
\hline 9 & $1: 2) C(=0) C$ \\
\hline 10 & $\mathrm{CC} 1 \mathrm{CC} 2 \mathrm{C} 3 \mathrm{CCC} 4=\mathrm{CC}(=0) \mathrm{C}=\mathrm{CC} 4(\mathrm{C}) \mathrm{C} 3 \mathrm{C}(0) \mathrm{CC} 2(\mathrm{C}) \mathrm{C} 1 \mathrm{C}(=\mathrm{O}) \mathrm{C}$ \\
\hline 11 & $\mathrm{CC}(=0) \mathrm{C} 1(0) \mathrm{CCC} 2 \mathrm{C} 3 \mathrm{CCC} 4=\mathrm{CC}(=0) \mathrm{CCC} 4(\mathrm{C}) \mathrm{C} 3 \mathrm{CCC} 12 \mathrm{C}$ \\
\hline 12 & $\mathrm{CCC}(=0) \mathrm{OC} 1 \mathrm{CCC} 2 \mathrm{C} 3 \mathrm{CCC} 4=\mathrm{CC}(=0) \mathrm{CCC} 4 \mathrm{C} 3 \mathrm{CCC} 12 \mathrm{C}$ \\
\hline 13 & $\mathrm{CC} 12 \mathrm{CCC} 3 \mathrm{C}(\mathrm{CCC} 4=\mathrm{CC}(=\mathrm{O}) \mathrm{C}=\mathrm{CC} 34 \mathrm{C}) \mathrm{C} 1 \mathrm{CCC} 2 \mathrm{C}=\mathrm{O}$ \\
\hline 14 & $\mathrm{CC} 12 \mathrm{CCC} 3 \mathrm{C}(\mathrm{CCC} 4=\mathrm{CC}(=0) \mathrm{CCC} 34) \mathrm{C} 1 \mathrm{CCC} 2(0) \mathrm{CHC}$ \\
\hline 15 & $\mathrm{CC} 1 \mathrm{CCC} 2 \mathrm{C} 3 \mathrm{CCC} 4=\mathrm{CC}(=0) \mathrm{CCC} 4(\mathrm{C}) \mathrm{C} 3 \mathrm{CCC} 12 \mathrm{C}$ \\
\hline 16 & $C \operatorname{coc}(=0) C 1=C(C) N C(=C(C 1 c 2: c: c: c: c: c: 2) C(=0) O C) C$ \\
\hline 17 & CC12CCC3C(CCc4:c:c(0):c:c:c3:4)C1CCC2O \\
\hline 18 & $\mathrm{CC} 12 \mathrm{CCC} 3 \mathrm{C}(\mathrm{CCC} 4=\mathrm{CCCCC} 34) \mathrm{C} 1 \mathrm{CCC} 2 \mathrm{O}$ \\
\hline 19 & c1:c:c:c(:c:c:1)C(c2:c:c:c:c:c:2)c3:c:c:c:c:c:3 \\
\hline 20 & $\mathrm{CN}(\mathrm{C}) \mathrm{C} 1 \mathrm{C} 2 \mathrm{CC} 3 \mathrm{C}(=\mathrm{C}(\mathrm{O}) \mathrm{C} 2(\mathrm{O}) \mathrm{C}(=\mathrm{O}) \mathrm{C}(=\mathrm{C} 10) \mathrm{C}(=\mathrm{O}) \mathrm{N}) \mathrm{C}(=0) \mathrm{c} 4: \mathrm{c}(\mathrm{O}): \mathrm{c}: \mathrm{c}: \mathrm{c}: \mathrm{c}: 4 \mathrm{C} 3(\mathrm{C}) \mathrm{O}$ \\
\hline 21 & $\mathrm{CC}(=0) \mathrm{C}=\mathrm{C} 1 \mathrm{CCC} 2 \mathrm{C} 3 \mathrm{CCCC} 3(\mathrm{C}) \mathrm{CcC} 2 \mathrm{C} 1(\mathrm{C}) \mathrm{C}$ \\
\hline 22 & $\operatorname{ccC} 1 \mathrm{CCC} 2 \mathrm{C}(\mathrm{CCC} 3(\mathrm{C}) \mathrm{C}(\mathrm{O}) \mathrm{CCC} 23) \mathrm{C} 1(\mathrm{C}) \mathrm{CC}$ \\
\hline 23 & $\operatorname{cc} 1 \mathrm{C}(\mathrm{CCC} 1(\mathrm{C}) \mathrm{O}) \mathrm{C} 2 \mathrm{CCC} 3=\mathrm{CC}(=\mathrm{O}) \mathrm{CCC} 3=\mathrm{C} 2 \mathrm{C}$ \\
\hline 24 & $C 1 C=C C(=C(c 2: c: c: c: c: c: 2) c 3: c: c: c: c: c: 3) C=C 1$ \\
\hline 25 & $\operatorname{CCC}(\mathrm{OC} 1 \mathrm{CC}(\mathrm{C})(\mathrm{O}) \mathrm{C}(\mathrm{OC}(=\mathrm{O}) \mathrm{C}) \mathrm{C}(\mathrm{C}) \mathrm{O} 1) \mathrm{C}(\mathrm{C}) \mathrm{O}$ \\
\hline 26 & CCc1:c:c(I):c(Oc2:c:c:c(O):c(I):c:2):c(I):c:1 \\
\hline 27 & $\operatorname{CCC}(\mathrm{C})(\mathrm{C}=\mathrm{C}) \mathrm{C} 1 \mathrm{CCC} 2(\mathrm{C}) \mathrm{C}(\mathrm{CCC} 2 \mathrm{C} 1 \mathrm{C}) \mathrm{C}=\mathrm{O}$ \\
\hline 28 & $\mathrm{CC}(\mathrm{C})(\mathrm{c} 1: \mathrm{c}: \mathrm{c}: \mathrm{c}(\mathrm{O}): \mathrm{c}: \mathrm{c}: 1) \mathrm{c} 2: \mathrm{c}: \mathrm{c}: \mathrm{c}(\mathrm{O}): \mathrm{c}: \mathrm{c}: 2$ \\
\hline 29 & $\mathrm{CCC}(0)(\mathrm{Cn} 1: \mathrm{c}: \mathrm{n}: \mathrm{c}: \mathrm{n}: 1) \mathrm{c} 2: \mathrm{c}: \mathrm{c}: \mathrm{c}(\mathrm{Cl}): \mathrm{c}: \mathrm{c}: 2$ \\
\hline
\end{tabular}




$\begin{array}{ll}30 & \text { CCCC1C(C)CCC2(C)C1CCC2(O)C\#C } \\ 31 & \text { CC(OC(=O)C)C(CCOC(=O)C)OC(=O)C } \\ 32 & \text { COc1:c:c:c(Cc2:c:c:c:c:c:2):c:c:1 } \\ 33 & \text { C(=Cc1:c:c:c:c:c:1)c2:c:c:c:c:c:2 } \\ 34 & \text { CCCN(CC)CCCc1:c:c:c:c:c:1 } \\ 35 & \text { O[Sn](c1:c:c:c:c:c:1)(c2:c:c:c:c:c:2)c3:c:c:c:c:c:3 } \\ 36 & \text { CCC(F)(F)C(F)(F)C(F)(F)C(F)(F)C(F)(F)C(F)(F)F } \\ 37 & \text { CCCCCCCC=CCCCCCCCN(C)CCO } \\ 38 & \text { CCCCCOCCC(C)OC1CC(O)CC(C)O1 } \\ 39 & \text { CCCCC(CC)COC(=O)c1:c:c:c:c:c:1 } \\ 40 & \text { Oc1:c:c:c(CCc2:c:c:c(O):c:c:2):c:c:1 } \\ 41 & \text { CCC(C)C1CCC(C)(C(C)O)C(C)C1CC } \\ 42 & \text { OCc1:c:c:c(Oc2:c:c:c:c:c:2):c:c:1 } \\ 43 & \text { Cc1:c:c:c:c:c:1N=Nc2:c:c:c:c:c:2 } \\ 44 & \text { CCN(c1:c:c:c:c:c:1)c2:c:c:c:c:c:2 } \\ 45 & \text { CC=C(N1CC(NC(=O)C)C1=O)C(=O)O } \\ 46 & \text { CCCCCCCCCCCOS(=O)=O } \\ 47 & \text { C(c1:c:c:c:c:c:1)c2:c:c:c:c:c:2 } \\ 48 & \text { CCCN(C)CCOc1:c:c:c:c:c:1 } \\ 49 & \text { c1ccc(cc1)Cc2ccccc2 } \\ 50 & \text { CCCN(C)c1:c:c:c:c:c:1CNC } \\ 51 & \text { CC1=CC(=O)Oc2:c:c:c:c:c1:2 } \\ 52 & \text { CC(CO)NC(=O)c1:c:c:c:c:c:1 } \\ 53 & \text { CCC(C)C(C)Cc1:c:c:c:c:c:1 } \\ 54 & \text { Oc1:c:c:c:c2:c:c:c:n:c:1:2 } \\ 55 & \text { CCCC(=O)Nc1:c:c:c:c:c:1 } \\ 56 & \text { CCCC(CC)CCCCC(C)C } \\ 57 & \text { CCCCCCCCCCOCC } \\ & \end{array}$

SI Table 1: SMILES strings for all structural alerts developed in this work. 


\section{Androgen Receptor}

Training Set

Test Set

\begin{tabular}{|c|c|c|c|c|c|c|c|c|c|c|c|c|c|c|c|c|}
\hline Alert No & TP & FN & TN & FP & SE & SP & $\mathbf{Q}$ & MCC & TP & FN & TN & FP & SE & SP & $\mathbf{Q}$ & MCC \\
\hline 1 & 17 & 661 & 4575 & 0 & 2.51 & 100.00 & 87.42 & 0.148 & 6 & 215 & 1550 & 3 & 2.71 & 99.81 & 87.71 & 0.117 \\
\hline 2 & 33 & 645 & 4574 & 1 & 4.87 & 99.98 & 87.70 & 0.203 & 8 & 213 & 1550 & 3 & 3.62 & 99.81 & 87.82 & 0.144 \\
\hline 3 & 16 & 662 & 4573 & 2 & 2.36 & 99.96 & 87.36 & 0.133 & 2 & 219 & 1553 & 0 & 0.90 & 100.00 & 87.66 & 0.089 \\
\hline 4 & 62 & 616 & 4571 & 4 & 9.14 & 99.91 & 88.20 & 0.273 & 13 & 208 & 1550 & 3 & 5.88 & 99.81 & 88.11 & 0.199 \\
\hline 5 & 36 & 642 & 4571 & 4 & 5.31 & 99.91 & 87.70 & 0.201 & 4 & 217 & 1553 & 0 & 1.81 & 100.00 & 87.77 & 0.126 \\
\hline 6 & 42 & 636 & 4568 & 7 & 6.19 & 99.85 & 87.76 & 0.211 & 15 & 206 & 1553 & 0 & 6.79 & 100.00 & 88.39 & 0.245 \\
\hline 7 & 93 & 585 & 4556 & 19 & 13.72 & 99.58 & 88.50 & 0.309 & 19 & 202 & 1546 & 7 & 8.60 & 99.55 & 88.22 & 0.224 \\
\hline 8 & 12 & 666 & 4561 & 14 & 1.77 & 99.69 & 87.06 & 0.070 & 0 & 221 & 1546 & 7 & 0.00 & 99.55 & 87.15 & -0.024 \\
\hline 9 & 12 & 666 & 4574 & 1 & 1.77 & 99.98 & 87.30 & 0.118 & 6 & 215 & 1552 & 1 & 2.71 & 99.94 & 87.82 & 0.140 \\
\hline 10 & 16 & 662 & 4562 & 13 & 2.36 & 99.72 & 87.15 & 0.094 & 4 & 217 & 1545 & 8 & 1.81 & 99.48 & 87.32 & 0.052 \\
\hline 11 & 11 & 667 & 4548 & 27 & 1.62 & 99.41 & 86.79 & 0.041 & 0 & 221 & 1544 & 9 & 0.00 & 99.42 & 87.03 & -0.027 \\
\hline 12 & 66 & 612 & 4424 & 151 & 9.73 & 96.70 & 85.47 & 0.108 & 19 & 202 & 1493 & 60 & 8.60 & 96.14 & 85.23 & 0.076 \\
\hline 13 & 10 & 668 & 4572 & 3 & 1.47 & 99.93 & 87.23 & 0.095 & 6 & 215 & 1553 & 0 & 2.71 & 100.00 & 87.88 & 0.154 \\
\hline 14 & 11 & 667 & 4566 & 9 & 1.62 & 99.80 & 87.13 & 0.078 & 0 & 221 & 1551 & 2 & 0.00 & 99.87 & 87.43 & -0.013 \\
\hline 15 & 15 & 663 & 4540 & 35 & 2.21 & 99.23 & 86.71 & 0.050 & 6 & 215 & 1542 & 11 & 2.71 & 99.29 & 87.26 & 0.068 \\
\hline 16 & 15 & 663 & 4535 & 40 & 2.21 & 99.13 & 86.62 & 0.044 & 3 & 218 & 1536 & 17 & 1.36 & 98.91 & 86.75 & 0.008 \\
\hline 17 & 66 & 612 & 4423 & 151 & 9.73 & 96.70 & 85.47 & 0.108 & 19 & 202 & 1493 & 60 & 8.60 & 96.14 & 85.23 & 0.076 \\
\hline 18 & 19 & 659 & 4529 & 46 & 2.80 & 98.99 & 86.58 & 0.054 & 6 & 215 & 1539 & 14 & 2.71 & 99.10 & 87.09 & 0.057 \\
\hline 19 & 122 & 556 & 4536 & 39 & 17.99 & 99.15 & 88.67 & 0.333 & 34 & 187 & 1545 & 8 & 15.38 & 99.48 & 89.01 & 0.323 \\
\hline 20 & 139 & 539 & 4457 & 118 & 20.50 & 97.42 & 87.49 & 0.279 & 39 & 182 & 1523 & 30 & 17.65 & 98.07 & 88.05 & 0.268 \\
\hline 21 & 26 & 652 & 4518 & 57 & 3.83 & 98.75 & 86.50 & 0.070 & 7 & 214 & 1530 & 23 & 3.17 & 98.52 & 86.64 & 0.043 \\
\hline 22 & 12 & 666 & 4517 & 58 & 1.77 & 98.73 & 86.22 & 0.015 & 3 & 218 & 1537 & 16 & 1.36 & 98.97 & 86.81 & 0.010 \\
\hline 23 & 11 & 667 & 4570 & 5 & 1.62 & 99.89 & 87.21 & 0.092 & 0 & 221 & 1551 & 2 & 0.00 & 99.87 & 87.43 & -0.013 \\
\hline 24 & 16 & 662 & 4485 & 90 & 2.36 & 98.03 & 85.68 & 0.009 & 3 & 218 & 1521 & 32 & 1.36 & 97.94 & 85.91 & -0.017 \\
\hline 25 & 15 & 663 & 4543 & 32 & 2.21 & 99.30 & 86.77 & 0.054 & 2 & 219 & 1541 & 12 & 0.90 & 99.23 & 86.98 & 0.005 \\
\hline 26 & 8 & 670 & 4557 & 18 & 1.18 & 99.61 & 86.90 & 0.038 & 2 & 219 & 1541 & 12 & 0.90 & 99.23 & 86.98 & 0.005 \\
\hline 27 & 8 & 670 & 4549 & 26 & 1.18 & 99.43 & 86.75 & 0.026 & 5 & 216 & 1542 & 11 & 2.26 & 99.29 & 87.20 & 0.054 \\
\hline 28 & 19 & 659 & 4488 & 87 & 2.80 & 98.10 & 85.80 & 0.021 & 7 & 214 & 1516 & 37 & 3.17 & 97.62 & 85.85 & 0.017 \\
\hline 29 & 15 & 663 & 4496 & 79 & 2.21 & 98.27 & 85.87 & 0.012 & 4 & 217 & 1527 & 26 & 1.81 & 98.33 & 86.30 & 0.003 \\
\hline 30 & 164 & 514 & 4390 & 185 & 24.19 & 95.96 & 86.69 & 0.271 & 50 & 171 & 1510 & 43 & 22.62 & 97.23 & 87.94 & 0.294 \\
\hline 31 & 8 & 670 & 4543 & 32 & 1.18 & 99.30 & 86.64 & 0.019 & 4 & 217 & 1545 & 8 & 1.81 & 99.48 & 87.32 & 0.052 \\
\hline
\end{tabular}




\begin{tabular}{|c|c|c|c|c|c|c|c|c|c|c|c|c|c|c|c|c|}
\hline 32 & 6 & 672 & 4569 & 6 & 0.88 & 99.87 & 87.09 & 0.053 & 0 & 221 & 1551 & 2 & 0.00 & 99.87 & 87.43 & -0.013 \\
\hline 33 & 63 & 615 & 4350 & 225 & 9.29 & 95.08 & 84.01 & 0.064 & 21 & 200 & 1461 & 92 & 9.50 & 94.08 & 83.54 & 0.048 \\
\hline 34 & 32 & 646 & 4410 & 165 & 4.72 & 96.39 & 84.56 & 0.020 & 8 & 213 & 1487 & 66 & 3.62 & 95.75 & 84.27 & -0.010 \\
\hline 35 & 15 & 663 & 4519 & 56 & 2.21 & 98.78 & 86.31 & 0.029 & 4 & 217 & 1537 & 16 & 1.81 & 98.97 & 86.87 & 0.024 \\
\hline 36 & 14 & 664 & 4528 & 47 & 2.06 & 98.97 & 86.46 & 0.032 & 7 & 214 & 1530 & 23 & 3.17 & 98.52 & 86.64 & 0.043 \\
\hline 37 & 7 & 671 & 4567 & 8 & 1.03 & 99.83 & 87.07 & 0.054 & 0 & 221 & 1550 & 3 & 0.00 & 99.81 & 87.37 & -0.016 \\
\hline 38 & 22 & 656 & 4397 & 178 & 3.24 & 96.11 & 84.12 & -0.011 & 8 & 213 & 1486 & 67 & 3.62 & 95.69 & 84.22 & -0.011 \\
\hline 39 & 25 & 653 & 4448 & 127 & 3.69 & 97.22 & 85.15 & 0.018 & 3 & 218 & 1499 & 54 & 1.36 & 96.52 & 84.67 & -0.040 \\
\hline 40 & 29 & 649 & 4427 & 148 & 4.28 & 96.77 & 84.83 & 0.019 & 6 & 215 & 1502 & 51 & 2.71 & 96.72 & 85.01 & -0.011 \\
\hline 41 & 19 & 659 & 4476 & 99 & 2.80 & 97.84 & 85.57 & 0.014 & 3 & 218 & 1525 & 28 & 1.36 & 98.20 & 86.13 & -0.011 \\
\hline 42 & 35 & 643 & 4394 & 181 & 5.16 & 96.04 & 84.31 & 0.020 & 10 & 211 & 1486 & 67 & 4.52 & 95.69 & 84.33 & 0.003 \\
\hline 43 & 30 & 648 & 4458 & 117 & 4.42 & 97.44 & 85.44 & 0.038 & 8 & 213 & 1513 & 40 & 3.62 & 97.42 & 85.74 & 0.021 \\
\hline 44 & 10 & 668 & 4497 & 78 & 1.47 & 98.30 & 85.80 & -0.006 & 1 & 220 & 1529 & 24 & 0.45 & 98.45 & 86.25 & -0.031 \\
\hline 45 & 15 & 663 & 4469 & 106 & 2.21 & 97.68 & 85.36 & -0.002 & 5 & 216 & 1504 & 49 & 2.26 & 96.84 & 85.06 & -0.017 \\
\hline 46 & 7 & 671 & 4525 & 50 & 1.03 & 98.91 & 86.27 & -0.002 & 2 & 219 & 1532 & 21 & 0.90 & 98.65 & 86.47 & -0.013 \\
\hline 47 & 49 & 629 & 4423 & 152 & 7.23 & 96.68 & 85.13 & 0.068 & 15 & 206 & 1493 & 60 & 6.79 & 96.14 & 85.01 & 0.048 \\
\hline 48 & 57 & 621 & 4285 & 290 & 8.41 & 93.66 & 82.66 & 0.028 & 11 & 210 & 1456 & 97 & 4.98 & 93.75 & 82.69 & -0.018 \\
\hline 49 & 7 & 671 & 4558 & 17 & 1.03 & 99.63 & 86.90 & 0.033 & 2 & 219 & 1548 & 5 & 0.90 & 99.68 & 87.37 & 0.031 \\
\hline 50 & 8 & 670 & 4552 & 23 & 1.18 & 99.50 & 86.81 & 0.030 & 1 & 220 & 1547 & 6 & 0.45 & 99.61 & 87.26 & 0.003 \\
\hline 51 & 142 & 536 & 4517 & 58 & 20.94 & 98.73 & 88.69 & 0.345 & 39 & 182 & 1535 & 18 & 17.65 & 98.84 & 88.73 & 0.309 \\
\hline 52 & 119 & 559 & 4013 & 562 & 17.55 & 87.72 & 78.66 & 0.053 & 23 & 198 & 1358 & 195 & 10.41 & 87.44 & 77.85 & -0.022 \\
\hline 53 & 3 & 675 & 4573 & 2 & 0.44 & 99.96 & 87.11 & 0.043 & 0 & 221 & 1553 & 0 & 0.00 & 100.00 & 87.54 & \#DIV/0! \\
\hline 54 & 5 & 673 & 4554 & 21 & 0.74 & 99.54 & 86.79 & 0.013 & 1 & 220 & 1550 & 3 & 0.45 & 99.81 & 87.43 & 0.018 \\
\hline 55 & 4 & 674 & 4559 & 16 & 0.59 & 99.65 & 86.86 & 0.013 & 0 & 221 & 1550 & 3 & 0.00 & 99.81 & 87.37 & -0.016 \\
\hline 56 & 6 & 672 & 4562 & 13 & 0.88 & 99.72 & 86.96 & 0.034 & 2 & 219 & 1548 & 5 & 0.90 & 99.68 & 87.37 & 0.031 \\
\hline 57 & 5 & 673 & 4535 & 40 & 0.74 & 99.13 & 86.43 & -0.005 & 3 & 218 & 1538 & 15 & 1.36 & 99.03 & 86.87 & 0.013 \\
\hline 58 & 103 & 575 & 3974 & 601 & 15.19 & 86.86 & 77.61 & 0.020 & 29 & 192 & 1340 & 213 & 13.12 & 86.28 & 77.17 & -0.006 \\
\hline 59 & 140 & 538 & 4430 & 145 & 20.65 & 96.83 & 87.00 & 0.259 & 40 & 181 & 1507 & 46 & 18.10 & 97.04 & 87.20 & 0.233 \\
\hline 60 & 24 & 654 & 4502 & 73 & 3.54 & 98.40 & 86.16 & 0.048 & 7 & 214 & 1522 & 31 & 3.17 & 98.00 & 86.19 & 0.027 \\
\hline 61 & 4 & 674 & 4533 & 42 & 0.59 & 99.08 & 86.37 & -0.012 & 2 & 219 & 1534 & 19 & 0.90 & 98.78 & 86.58 & -0.010 \\
\hline 62 & 43 & 635 & 4356 & 219 & 6.34 & 95.21 & 83.74 & 0.024 & 13 & 208 & 1477 & 76 & 5.88 & 95.11 & 83.99 & 0.015 \\
\hline 63 & 3 & 675 & 4569 & 6 & 0.44 & 99.87 & 87.04 & 0.025 & 0 & 221 & 1551 & 2 & 0.00 & 99.87 & 87.43 & -0.013 \\
\hline 64 & 13 & 665 & 4536 & 39 & 1.92 & 99.15 & 86.60 & 0.036 & 8 & 213 & 1544 & 9 & 3.62 & 99.42 & 87.49 & 0.103 \\
\hline 65 & 35 & 643 & 4443 & 132 & 5.16 & 97.11 & 85.25 & 0.044 & 2 & 219 & 1486 & 67 & 0.90 & 95.69 & 83.88 & -0.058 \\
\hline
\end{tabular}




\begin{tabular}{|c|c|c|c|c|c|c|c|c|c|c|c|c|c|c|c|c|}
\hline 66 & 313 & 365 & 2818 & 1757 & 46.17 & 61.60 & 59.60 & 0.053 & 96 & 125 & 917 & 636 & 43.44 & 59.05 & 57.10 & 0.017 \\
\hline 67 & 2 & 676 & 4575 & 0 & 0.29 & 100.00 & 87.13 & 0.051 & 0 & 221 & 1553 & 0 & 0.00 & 100.00 & 87.54 & \#DIV/0! \\
\hline 69 & 2 & 676 & 4575 & 0 & 0.29 & 100.00 & 87.13 & 0.051 & 1 & 220 & 1553 & 0 & 0.45 & 100.00 & 87.60 & 0.063 \\
\hline 71 & 2 & 676 & 4574 & 1 & 0.29 & 99.98 & 87.11 & 0.038 & 0 & 221 & 1553 & 0 & 0.00 & 100.00 & 87.54 & \#DIV/0! \\
\hline 72 & 14 & 664 & 4544 & 31 & 2.06 & 99.32 & 86.77 & 0.050 & 3 & 218 & 1536 & 17 & 1.36 & 98.91 & 86.75 & 0.008 \\
\hline 73 & 2 & 676 & 4574 & 1 & 0.29 & 99.98 & 87.11 & 0.038 & 0 & 221 & 1553 & 0 & 0.00 & 100.00 & 87.54 & \#DIV/0! \\
\hline 76 & 2 & 676 & 4574 & 1 & 0.29 & 99.98 & 87.11 & 0.038 & 0 & 221 & 1553 & 0 & 0.00 & 100.00 & 87.54 & \#DIV/0! \\
\hline 77 & 15 & 663 & 4518 & 57 & 2.21 & 98.75 & 86.29 & 0.028 & 11 & 210 & 1534 & 19 & 4.98 & 98.78 & 87.09 & 0.096 \\
\hline 78 & 8 & 670 & 4546 & 29 & 1.18 & 99.37 & 86.69 & 0.022 & 2 & 219 & 1548 & 5 & 0.90 & 99.68 & 87.37 & 0.031 \\
\hline 79 & 205 & 473 & 4083 & 492 & 30.24 & 89.25 & 81.63 & 0.193 & 65 & 156 & 1403 & 150 & 29.41 & 90.34 & 82.75 & 0.200 \\
\hline 80 & 2 & 676 & 4570 & 5 & 0.29 & 99.89 & 87.04 & 0.017 & 0 & 221 & 1552 & 1 & 0.00 & 99.94 & 87.49 & -0.009 \\
\hline 81 & 32 & 646 & 4456 & 119 & 4.72 & 97.40 & 85.44 & 0.043 & 6 & 215 & 1516 & 37 & 2.71 & 97.62 & 85.79 & 0.007 \\
\hline 86 & 6 & 672 & 4543 & 32 & 0.88 & 99.30 & 86.60 & 0.007 & 2 & 219 & 1542 & 11 & 0.90 & 99.29 & 87.03 & 0.008 \\
\hline 87 & 6 & 672 & 4540 & 35 & 0.88 & 99.23 & 86.54 & 0.005 & 1 & 220 & 1541 & 12 & 0.45 & 99.23 & 86.92 & -0.012 \\
\hline
\end{tabular}

Glucocorticoid Receptor

\begin{tabular}{|c|c|c|c|c|c|c|c|c|c|c|c|c|c|c|c|c|}
\hline \multirow[b]{2}{*}{ Alert No } & \multicolumn{8}{|c|}{ Training Set } & \multicolumn{8}{|c|}{ Test Set } \\
\hline & TP & FN & TN & FP & SE & SP & $\mathbf{Q}$ & MCC & TP & FN & TN & FP & SE & SP & $\mathbf{Q}$ & MCC \\
\hline 1 & 5 & 244 & 5228 & 0 & 2.01 & 100.00 & 95.55 & 0.139 & 2 & 72 & 1778 & 0 & 2.70 & 100.00 & 96.11 & 0.161 \\
\hline 2 & 9 & 240 & 5228 & 0 & 3.61 & 100.00 & 95.62 & 0.186 & 5 & 69 & 1778 & 0 & 6.76 & 100.00 & 96.27 & 0.255 \\
\hline 3 & 16 & 233 & 5228 & 0 & 6.43 & 100.00 & 95.75 & 0.248 & 7 & 67 & 1778 & 0 & 9.46 & 100.00 & 96.38 & 0.302 \\
\hline 4 & 6 & 243 & 5228 & 0 & 2.41 & 100.00 & 95.56 & 0.152 & 3 & 71 & 1778 & 0 & 4.05 & 100.00 & 96.17 & 0.197 \\
\hline 5 & 29 & 220 & 5228 & 0 & 11.65 & 100.00 & 95.98 & 0.334 & 12 & 62 & 1778 & 0 & 16.22 & 100.00 & 96.65 & 0.396 \\
\hline 6 & 5 & 244 & 5228 & 0 & 2.01 & 100.00 & 95.55 & 0.139 & 3 & 71 & 1778 & 0 & 4.05 & 100.00 & 96.17 & 0.197 \\
\hline 7 & 6 & 243 & 5228 & 0 & 2.41 & 100.00 & 95.56 & 0.152 & 2 & 72 & 1777 & 1 & 2.70 & 99.94 & 96.06 & 0.129 \\
\hline 8 & 6 & 243 & 5228 & 0 & 2.41 & 100.00 & 95.56 & 0.152 & 0 & 74 & 1778 & 0 & 0.00 & 100.00 & 96.00 & \#DIV/0! \\
\hline
\end{tabular}




\begin{tabular}{|c|c|c|c|c|c|c|c|c|c|c|c|c|c|c|c|c|}
\hline 9 & 4 & 245 & 5228 & 0 & 1.61 & 100.00 & 95.53 & 0.124 & 0 & 74 & 1778 & 0 & 0.00 & 100.00 & 96.00 & \#DIV/0! \\
\hline 11 & 13 & 236 & 5227 & 1 & 5.22 & 99.98 & 95.67 & 0.215 & 3 & 71 & 1778 & 0 & 4.05 & 100.00 & 96.17 & 0.197 \\
\hline 12 & 8 & 241 & 5226 & 2 & 3.21 & 99.96 & 95.56 & 0.155 & 2 & 72 & 1776 & 2 & 2.70 & 99.89 & 96.00 & 0.109 \\
\hline 13 & 41 & 208 & 5228 & 0 & 16.47 & 100.00 & 96.20 & 0.398 & 15 & 59 & 1778 & 0 & 20.27 & 100.00 & 96.81 & 0.443 \\
\hline 14 & 4 & 245 & 5228 & 0 & 1.61 & 100.00 & 95.53 & 0.124 & 1 & 73 & 1778 & 0 & 1.35 & 100.00 & 96.06 & 0.114 \\
\hline 15 & 20 & 229 & 5226 & 2 & 8.03 & 99.96 & 95.78 & 0.263 & 6 & 68 & 1777 & 1 & 8.11 & 99.94 & 96.27 & 0.257 \\
\hline 16 & 4 & 245 & 5226 & 2 & 1.61 & 99.96 & 95.49 & 0.099 & 1 & 73 & 1777 & 1 & 1.35 & 99.94 & 96.00 & 0.077 \\
\hline 17 & 8 & 241 & 5226 & 2 & 3.21 & 99.96 & 95.56 & 0.155 & 0 & 74 & 1776 & 2 & 0.00 & 99.89 & 95.90 & -0.007 \\
\hline 19 & 4 & 245 & 5221 & 7 & 1.61 & 99.87 & 95.40 & 0.069 & 2 & 72 & 1775 & 3 & 2.70 & 99.83 & 95.95 & 0.096 \\
\hline 20 & 3 & 246 & 5222 & 6 & 1.20 & 99.89 & 95.40 & 0.056 & 0 & 74 & 1778 & 0 & 0.00 & 100.00 & 96.00 & \#DIV/O! \\
\hline 21 & 68 & 181 & 5224 & 4 & 27.31 & 99.92 & 96.62 & 0.498 & 22 & 52 & 1776 & 2 & 29.73 & 99.89 & 97.08 & 0.513 \\
\hline 22 & 3 & 246 & 5222 & 6 & 1.20 & 99.89 & 95.40 & 0.056 & 0 & 74 & 1776 & 2 & 0.00 & 99.89 & 95.90 & -0.007 \\
\hline 23 & 3 & 246 & 5228 & 0 & 1.20 & 100.00 & 95.51 & 0.107 & 0 & 74 & 1778 & 0 & 0.00 & 100.00 & 96.00 & \#DIV/O! \\
\hline 24 & 3 & 246 & 5225 & 3 & 1.20 & 99.94 & 95.45 & 0.072 & 0 & 74 & 1778 & 0 & 0.00 & 100.00 & 96.00 & \#DIV/0! \\
\hline 29 & 3 & 246 & 5228 & 0 & 1.20 & 100.00 & 95.51 & 0.107 & 0 & 74 & 1778 & 0 & 0.00 & 100.00 & 96.00 & \#DIV/0! \\
\hline 30 & 10 & 239 & 5228 & 0 & 4.02 & 100.00 & 95.64 & 0.196 & 1 & 73 & 1777 & 1 & 1.35 & 99.94 & 96.00 & 0.077 \\
\hline 31 & 3 & 246 & 5225 & 3 & 1.20 & 99.94 & 95.45 & 0.072 & 0 & 74 & 1777 & 1 & 0.00 & 99.94 & 95.95 & -0.005 \\
\hline 32 & 3 & 246 & 5206 & 22 & 1.20 & 99.58 & 95.11 & 0.024 & 1 & 73 & 1769 & 9 & 1.35 & 99.49 & 95.57 & 0.023 \\
\hline 33 & 4 & 245 & 5213 & 15 & 1.61 & 99.71 & 95.25 & 0.047 & 2 & 72 & 1771 & 7 & 2.70 & 99.61 & 95.73 & 0.065 \\
\hline 34 & 3 & 246 & 5180 & 48 & 1.20 & 99.08 & 94.63 & 0.006 & 0 & 74 & 1765 & 13 & 0.00 & 99.27 & 95.30 & -0.017 \\
\hline 35 & 2 & 247 & 5228 & 0 & 0.80 & 100.00 & 95.49 & 0.088 & 0 & 74 & 1778 & 0 & 0.00 & 100.00 & 96.00 & \#DIV/0! \\
\hline 36 & 2 & 247 & 5219 & 9 & 0.80 & 99.83 & 95.33 & 0.029 & 0 & 74 & 1776 & 2 & 0.00 & 99.89 & 95.90 & -0.007 \\
\hline 37 & 2 & 247 & 5227 & 1 & 0.80 & 99.98 & 95.47 & 0.070 & 0 & 74 & 1778 & 0 & 0.00 & 100.00 & 96.00 & \#DIV/0! \\
\hline 38 & 3 & 246 & 5213 & 15 & 1.20 & 99.71 & 95.23 & 0.033 & 0 & 74 & 1775 & 3 & 0.00 & 99.83 & 95.84 & -0.008 \\
\hline 39 & 2 & 247 & 5220 & 8 & 0.80 & 99.85 & 95.34 & 0.032 & 0 & 74 & 1777 & 1 & 0.00 & 99.94 & 95.95 & -0.005 \\
\hline 40 & 2 & 247 & 5214 & 14 & 0.80 & 99.73 & 95.23 & 0.021 & 0 & 74 & 1776 & 2 & 0.00 & 99.89 & 95.90 & -0.007 \\
\hline
\end{tabular}




$\begin{array}{ccccccccccccccccc}43 & 2 & 247 & 5227 & 1 & 0.80 & 99.98 & 95.47 & 0.070 & 1 & 73 & 1776 & 2 & 1.35 & 99.89 & 95.95 & 0.060 \\ 44 & 2 & 247 & 5182 & 46 & 0.80 & 99.12 & 94.65 & -0.002 & 0 & 74 & 1771 & 7 & 0.00 & 99.61 & 95.63 & -0.013 \\ 45 & 2 & 247 & 5205 & 23 & 0.80 & 99.56 & 95.07 & 0.011 & 0 & 74 & 1767 & 11 & 0.00 & 99.38 & 95.41 & -0.016 \\ 46 & 2 & 247 & 5228 & 0 & 0.80 & 100.00 & 95.49 & 0.088 & 1 & 73 & 1777 & 1 & 1.35 & 99.94 & 96.00 & 0.077 \\ 47 & 24 & 225 & 5015 & 213 & 9.64 & 95.93 & 92.00 & 0.057 & 10 & 64 & 1692 & 86 & 13.51 & 95.16 & 91.90 & 0.077 \\ 48 & 2 & 247 & 5220 & 8 & 0.80 & 99.85 & 95.34 & 0.032 & 0 & 74 & 1776 & 2 & 0.00 & 99.89 & 95.90 & -0.007 \\ 49 & 24 & 225 & 5014 & 213 & 9.64 & 95.93 & 92.00 & 0.057 & 10 & 64 & 1692 & 86 & 13.51 & 95.16 & 91.90 & 0.077 \\ 50 & 2 & 247 & 5228 & 0 & 0.80 & 100.00 & 95.49 & 0.088 & 0 & 74 & 1778 & 0 & 0.00 & 100.00 & 96.00 & \# D I V / 0 ! \\ 51 & 2 & 247 & 5223 & 5 & 0.80 & 99.90 & 95.40 & 0.041 & 1 & 73 & 1775 & 3 & 1.35 & 99.83 & 95.90 & 0.050 \\ 52 & 2 & 247 & 5219 & 9 & 0.80 & 99.83 & 95.33 & 0.029 & 0 & 74 & 1775 & 3 & 0.00 & 99.83 & 95.84 & -0.008 \\ 53 & 11 & 238 & 5215 & 13 & 4.42 & 99.75 & 95.42 & 0.131 & 0 & 74 & 1773 & 5 & 0.00 & 99.72 & 95.73 & -0.011 \\ 54 & 2 & 247 & 5222 & 6 & 0.80 & 99.89 & 95.38 & 0.038 & 0 & 74 & 1775 & 3 & 0.00 & 99.83 & 95.84 & -0.008 \\ 55 & 3 & 246 & 5185 & 43 & 1.20 & 99.18 & 94.72 & 0.009 & 0 & 74 & 1767 & 11 & 0.00 & 99.38 & 95.41 & -0.016 \\ 56 & 109 & 140 & 5172 & 56 & 43.78 & 98.93 & 96.42 & 0.520 & 26 & 48 & 1751 & 27 & 35.14 & 98.48 & 95.95 & 0.395 \\ 57 & 57 & 192 & 5141 & 87 & 22.89 & 98.34 & 94.91 & 0.276 & 16 & 58 & 1738 & 40 & 21.62 & 97.75 & 94.71 & 0.222\end{array}$

SI Table 2: Statistical performance data and associated quantitative prediction data for all structural alerts developed in this work. In each case statistical performance data is calculated as outlined in the paper for both the training and test sets. TP=True Positive, FN=False Negative, TN=True Negative, FP=False Positive, SE=Sensitivity, SP=Specificity, Q=Overall Quality, MCC=Matthews Correlation Coefficient, N/A=not applicable . 